\title{
Experimental study of evaporation of sessile water droplet on PDMS surfaces
}

\author{
Ying-Song Yu $\cdot$ Zi-Qian Wang $\cdot$ Ya-Pu Zhao
}

Received: 8 July 2013 / Revised: 26 August 2013 / Accepted: 29 October 2013

(C)The Chinese Society of Theoretical and Applied Mechanics and Springer-Verlag Berlin Heidelberg 2013

\begin{abstract}
Evaporation of sessile water droplet on polydimethylsiloxane (PDMS) surfaces with three different curing ratios $(5: 1,10: 1$, and $20: 1)$ was experimentally investigated in this paper. We show that the constant contact radius (CCR) evaporation on surface with high curing ratio lasts longer than that with low curing ratio. We also measured Young's moduli of PDMS films by using atomic force microscopy (AFM) and simulated surface deformation of PDMS films induced by sessile water droplet. With increasing curing ratio of PDMS film, Young's modulus of PDMS film is getting lower, and then there will be larger surface deformation and more elastic stored energy. Since such energy acts as a barrier to keep the three-phase contact line pinned, thus it will result in longer CCR evaporation on PDMS surface with higher curing ratio.
\end{abstract}

Keywords Droplet evaporation - PDMS - Constant contact radius mode . Constant contact angle mode - Forcedisplacement curve $\cdot$ Surface deformation · Liquid-vapor interfacial tension

The project was supported by the National Natural Science Foundation of China (11002051, 11072244, and 11372313), the Key Research Program of the Chinese Academy of Sciences (KJZDEW-M01), and the Instrument Developing Project of the Chinese Academy of Sciences (Y2010031).

\section{Y.-S. Yu}

Department of Engineering Mechanics,

School of Civil Engineering \& Architecture,

Hubei University of Technology, 430068 Wuhan, China

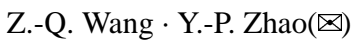

State Key Laboratory of Nonlinear Mechanics,

Institute of Mechanics, Chinese Academy of Sciences,

100190 Beijing, China

e-mail: yzhao@imech.ac.cn

\section{Introduction}

Since polydimethylsiloxane (PDMS) has many advantages such as good biocompatibility, nontoxicity and optical transparence, and better fabricability [1], it has been widely used in microelectromechnical systems (MEMS), micro total analysis system ( $\mu$-TAS) and lab-on-a-chip [2-4]. Because these devices made of PDMS are normally exposed to external environment, liquid in these devices will spontaneously evaporate and thus the working performance of these devices may be affected. Therefore, investigation on evaporation of water droplet on PDMS surfaces is of crucial importance in many research fields.

Droplet evaporation has found wide applications such as spray cooling, inkjet printing, coffee-ring effect, biological applications and so on [5-9]. In 1977, Picknett and Bexon [10] presented two different evaporation modes, that is, constant contact radius (CCR) mode and constant contact angle (CCA) mode. When water droplet evaporates in the CCR mode, attributing to contact angle hysteresis (CAH) [11] (Fig. 1a) which is mainly associated with line tension $[12,13]$, there is a pinning force per unit length acting at the three-phase contact line in the lateral direction

$f_{\text {pin }}=\gamma_{\mathrm{lv}}\left(\cos \theta_{\mathrm{r}}-\cos \theta_{\mathrm{a}}\right)$,

where $\gamma_{\mathrm{lv}}$ is the liquid-vapor interfacial tension, as shown in Fig. $1 \mathrm{~b}, \theta_{\mathrm{a}}$ and $\theta_{\mathrm{r}}$ are the advancing and receding contact angles, respectively. Such a force will make the contact line pinned and thus the contact radius is unaltered while the contact angle decreases with time. Moreover, the larger the $\mathrm{CAH}$, the stronger the pinning interaction of substrate to droplet and the larger the resistance to stop the droplet moving, therefore, large CAH is the key to coffee-ring effect [6]. When water droplet evaporates in the CCA mode, the contact angle is unaltered while the contact radius varies with time. From then on, lots of investigations have been reported on CCA and CCR evaporation, as summarized in Table 1. Besides, it should be noted that since there is a logarithmical 
Table 1 Investigations of CCR and CCA evaporation

\begin{tabular}{|c|c|c|c|}
\hline Authors & Substrate & Liquid & Description \\
\hline $\begin{array}{l}\text { Picknett \& } \\
\text { Bexon [10] }\end{array}$ & $\begin{array}{l}\text { Polytetrafluoroethylene } \\
\text { (PTFE) }\end{array}$ & Methyl acetoacetate & $\begin{array}{l}\text { First presentation of two evaporation modes and } \\
\text { theoretical solution for CCA evaporation }\end{array}$ \\
\hline McHale et al. [14] & Teflon & Water & \\
\hline Erbil et al. [15] & Teflon & $\begin{array}{l}n \text {-butanol, toluene, } n \text {-octane } \\
\text { and } n \text {-nonane }\end{array}$ & Theoretical solution for CCA evaporation \\
\hline $\begin{array}{l}\text { Bourgès-Monnier } \\
\& \text { Shanahan [16] }\end{array}$ & $\begin{array}{l}\text { Epoxy resin, polyethylene, } \\
\text { PTFE, glass }\end{array}$ & Water, $n$-decane & Theoretical analysis of CCR evaporation \\
\hline Rowan et al. [17] & Polymethyl methacrylate & Water & Solution for CCR evaporation $\left(\theta<90^{\circ}\right)$ \\
\hline McHale et al. [18] & Textured SU-8 & Water & Fitting solution for CCR evaporation \\
\hline Yu et al. [19] & PDMS, Teflon & Water & Theoretical solution for CCR evaporation \\
\hline Chen et al. [13] & Textured silicon & Water & $\begin{array}{l}\text { General models for predicting } \\
\text { the CCR-CCA transition }\end{array}$ \\
\hline Hao et al. [20] & Octadecyltrichorosilane & Water & $\begin{array}{l}\text { Influence of the scale of micropillars and the volume } \\
\text { of waterdroplets on the evaporation behaviors }\end{array}$ \\
\hline
\end{tabular}

singularity of thermal dissipation rate at the three-phase contact line, there is also a paradox in droplet evaporation, that is, "Not even Helios could evaporate a water droplet" [21]. As PDMS has been widely used in many fields, evaporation of water droplet on PDMS surfaces has been extensively investigated [19, 22-24]. Three different stages, namely CCR, CCA and mixed-mode evaporation are found in evaporation of water droplet on smooth PDMS surface [19, 22].

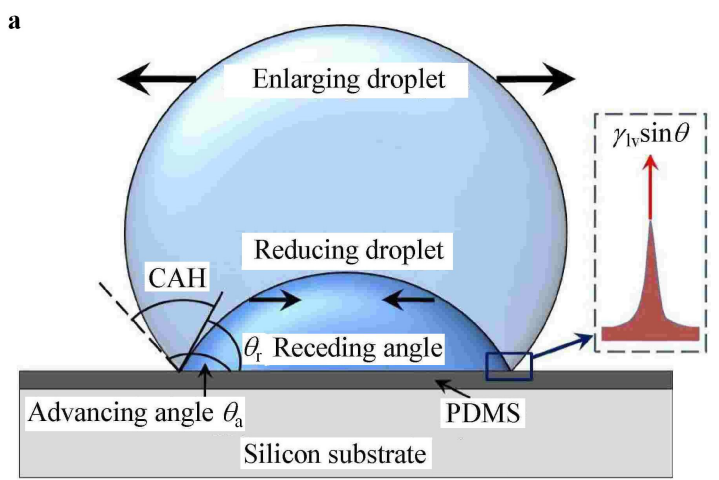

b

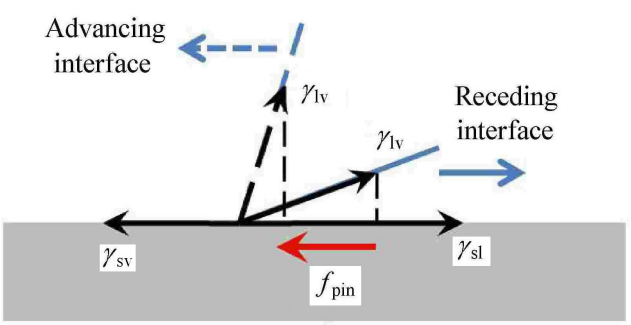

Fig. 1 Schematics of $\mathrm{CAH}$ and ridge-like surface deformation
It is known that when there is a sessile liquid droplet on a solid surface, there will be a ridge-like surface deformation (Fig. 1a) under the action of vertical component of liquid-vapor interfacial tension and Laplacian pressure [2529]. Such a deformation is found to be proportional to $\left(\gamma_{\mathrm{lv}} \sin \theta\right) / E$, with $\theta$ being the contact angle and $E$ being the Young's modulus of the solid. For materials whose Young's moduli are of the order of GPa or even larger, the maximum of the surface deformation is obviously less than $1 \mathrm{~nm}$ and can be neglected. However, for soft materials such as PDMS, the maximum of surface deformation is about several tens nanometers or even higher, and thus can no longer be readily neglected. The deformation may be associated with molecular orientation of the solid molecules at the three phase contact line $[12,30]$. The molecular orientation will result in a stronger solid-liquid intermolecular interaction, and hence stronger pinning. Recently, Lopes and Bonaccurso [31] experimentally investigated evaporation of sessile water drops on soft viscoelastic substrates, found that water drops on soft surfaces evaporated faster than those on hard surfaces and suggested that the faster evaporation rates on softer surfaces resulted from the substrate deformation close to the triplephase contact line.

However, up to now, to our best knowledge there have few reports on the effect of surface deformation on droplet evaporation. Therefore, in order to study the effect of surface deformation on droplet evaporation, we conducted evaporation experiments of sessile water droplet on PDMS surface and simulated surface deformation of PDMS surface induced by sessile water droplet in this paper. We think this work will help us to better understand the evaporation of water droplet on PDMS surface. 


\section{Experiments}

\subsection{Droplet evaporation}

We prepared PDMS films for studying droplet evaporation. PDMS (mass ratios of base to curing are $5: 1,10: 1$, and $20: 1$, Sylgard 184, Dow Corning, USA) was spin-coated onto the surface of clean silicon surface at the rate of about $1000 \mathrm{r} / \mathrm{min}$, then the samples were solidified in $150^{\circ} \mathrm{C}$ for 20 minutes. The thickness of PDMS was about $70 \mu \mathrm{m}$ [32]. To reduce the environmental effect on droplet evaporation, a chamber $(10.0 \mathrm{~cm} \times 10.0 \mathrm{~cm} \times 3.0 \mathrm{~cm})$ with a small hole at its top was used. Water droplet with volume of $3.0 \mu \mathrm{l}$ was generated by a syringe passing through the hole and deposited on sample surface. OCA 20 system (precision: $\pm 0.1^{\circ}$, from Dataphysics, Germany) equipped with a highresolution camera was used to record droplet evaporation with five seconds per frame, as shown in Fig. 2. The environmental temperature and relative humidity are $(24.3 \pm 1)^{\circ} \mathrm{C}$ and $(15 \pm 1) \%$, respectively. As soon as water droplet was deposited on the surfaces, the OCA 20 system was immediately adjusted to record the evaporation processes. To ensure the reproducibility, every experiment was repeated four times. Images of the water droplets were extracted with certain time interval from the videos. As an example, Fig. 3 illustrates the evaporation process of sessile water droplet on 20:1 PDMS surface, which will be discussed in the latter part.

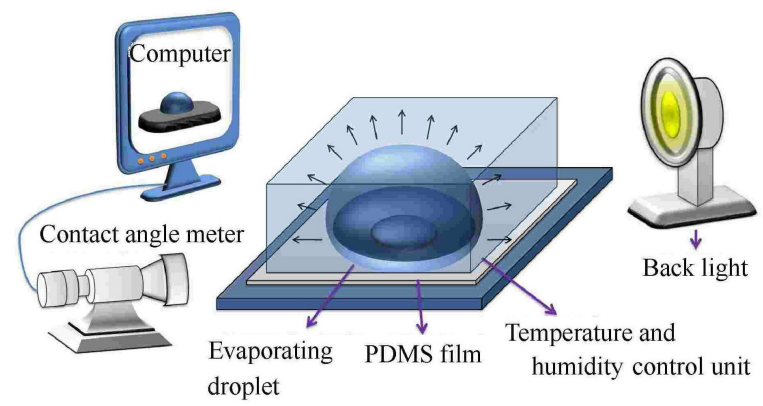

Fig. 2 Experimental schematics of water droplet evaporating on PDMS films

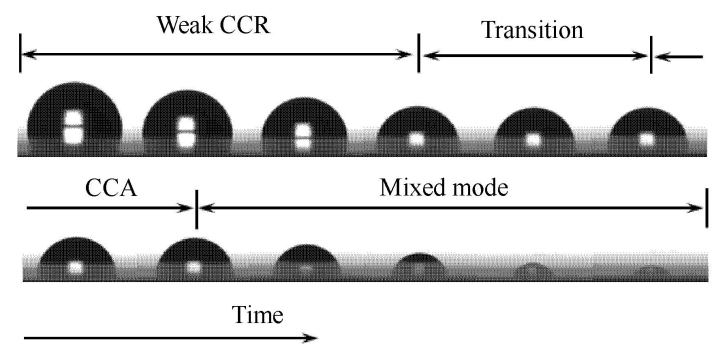

Fig. 3 Evaporation images of sessile water droplet on 20: 1 PDMS surface

\subsection{Force-displacement curves by AFM}

Recently, Chen et al. [33] used a piezo-rheometer to measure the shear modulus of PDMS films. In this paper, AFM is used to measure the Young's modulus of PDMS films. Johnson-Kendall-Roberts (JKR) theory [34], a model for soft deformable materials with strong adhesion energies, is used to analyze the force-displacement curves given by AFM. The curvature radius of the AFM tip is $20.0 \mathrm{~nm}$. The Poisson's ratio of AFM tip is $v_{1}=0.3$. The spring constant of the AFM cantilever is $0.12 \mathrm{~N} / \mathrm{m}$. Every piece of PDMS films was sampled twice and the force-displacement curves of PDMS films with different mass ratios are given in Fig. 4. The Young's modulus can be obtained from the curves by the following formula [34]

$E=\frac{3\left(1-v_{1}^{2}\right) P_{1}}{8}\left(\frac{3}{r_{\mathrm{m}} \Lambda_{0}^{3}}\right)^{\frac{1}{2}}$

where $r_{\mathrm{m}}=r_{1} r_{2} /\left(r_{1}+r_{2}\right), r_{1}$ and $r_{2}$ are the curvature radii of PDMS films and AFM tip, respectively. Since PDMS films are flat, $r_{\mathrm{m}}$ is $20.0 \mathrm{~nm} . P_{1}$ is the external force where there is no indentation in the sample, $\Lambda_{0}$ is the deformation which is induced by adhesion force only. $P_{1}$ and $\Lambda_{0}$ can be obtained from Fig. 4 and then Young's moduli of PDMS films were calculated and given in Table 2, which indicates that the higher the mass ratio of PDMS film, the smaller its Young's modulus.

Table 2 Young's modulus of PDMS films

\begin{tabular}{llll}
\hline Mass ratio & $5: 1$ & $10: 1$ & $20: 1$ \\
\hline$E / \mathrm{MPa}$ & 1.50 & 1.36 & 0.72 \\
\hline
\end{tabular}

\section{Surface deformation}

As we know, when there is a sessile liquid droplet on solid surface, the surface will be deformed under the action of vertical component of liquid-vapor interfacial tension and Laplacian pressure, and the vertical displacement at the surface is given as [19]

$$
\begin{aligned}
u_{z}(r, 0)= & \frac{4\left(1-v^{2}\right)}{E} \int_{0}^{\infty} f(\xi h) \frac{\mathrm{J}_{0}(\xi r)}{\xi} \\
& \times\left[R_{1} \tau \mathrm{J}_{1}\left(\xi R_{1}\right)-R(\Delta P+\tau) \mathrm{J}_{1}(\xi R)\right] \mathrm{d} \xi,
\end{aligned}
$$

where

$$
\begin{array}{ll}
f(x)=\frac{\sinh ^{2} x}{2 x+\sinh 2 x}, & \Delta P=\frac{2 \gamma_{\mathrm{lv}} \sin \theta}{R}, \\
\tau=\frac{\gamma_{\mathrm{lv}} \sin \theta}{\delta}, & R_{1}=R+\delta,
\end{array}
$$

here $R$ is the contact radius, $\delta$ is the width of liquid-vapor interfacial layer, and $h$ is the thickness of the solid film. $\mathbf{J}_{0}$ and $J_{1}$ are Bessel functions of orders zero and one, respectively. For PDMS is a rubber-like hyperelastic material, its Poisson's ratio $v$ is approximately 0.5. Equation (3) gives the surface deformation induced by sessile water droplet, however, it is difficult for integration. Therefore, in this part, we used ANSYS to calculate the surface deformation. Figure 1a 

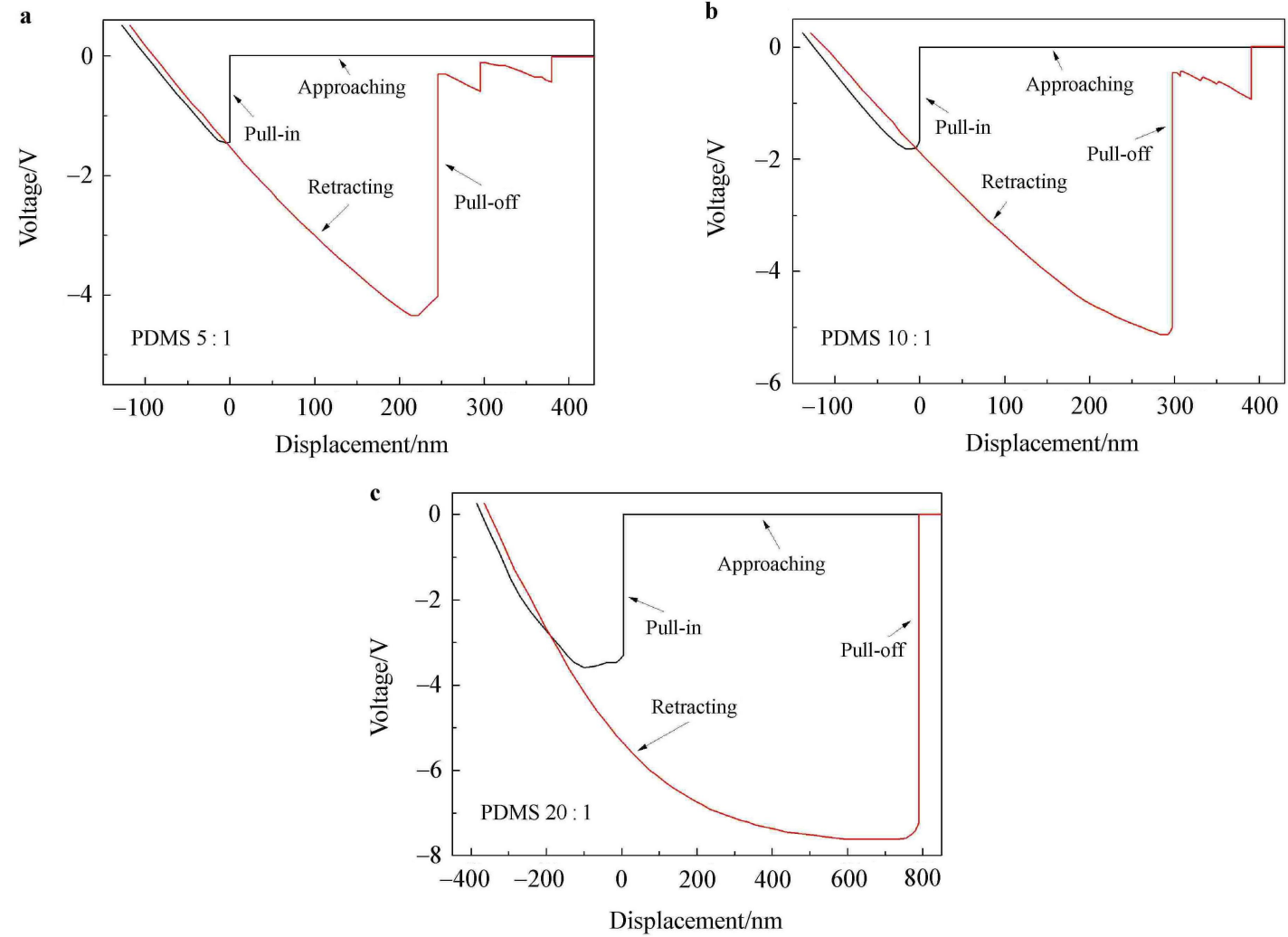

Fig. 4 Force-displacement curves of PDMS film. a 5 : 1 ; b $10: 1$; c $20: 1$

shows a sessile water droplet on thin PDMS film which is adhered to clean silicon substrate. The thickness of PDMS film is set to be $70.0 \mu \mathrm{m}$. The radius of circular PDMS film is set to be $10.0 \mathrm{~mm}$. On the top surface of PDMS films, there are vertical component of liquid-vapor interfacial tension acting at the contact line and Laplacian pressure acting on the area where the droplet meets with the film. The Poisson's ratio is set to be $v=0.499$. In numerical simulations, about 85400 elements with 97608 nodes are used.

\section{Results and discussion}

Figures $5 \mathrm{a}$ and $5 \mathrm{~b}$ show the normalized contact radius $R(t) / R_{0}\left(R_{0}\right.$ is the initial contact radius and $R(t)$ the instantaneous contact radius.) and the instantaneous contact angle $\theta(t)$ vs. time $t$ for $3.0 \mu \mathrm{l}$ water droplet on PDMS films with different mass ratios, respectively. When $3.0 \mu \mathrm{l}$ water droplet evaporates on 5: 1 PDMS surface, in about the first $150 \mathrm{~s}$, the contact radius is unaltered while the contact angle decreases from $105.46^{\circ}$ to $99.83^{\circ}$. This stage is called as CCR evaporation. Then there is a short time transition, during this transition, the contact radius decreases while the contact angle increases. Such a transition was called as stick-slip behavior. Similar phenomenon was also found in the evaporation of ethanol droplets on Teflon [35], and Shanahan and Sefiane [35] set up a simple model using excess free energy to explain the stick-slip behavior. After the transition, the contact radius is altered while the contact angle is almost unal- tered for about $475 \mathrm{~s}$, therefore, we thought that the droplet now evaporates in the CCA mode. When this stage is over, both the contact radius and the contact angle are changed, and this stage is called as mixed-mode evaporation. Similar to evaporation of water droplet on 5:1 PDMS surface, the evaporation of water droplet on 10:1 PDMS surface can also be divided into four stages. In the first stage, the contact radius is unaltered for a longer time-about $350 \mathrm{~s}$. After a short time transition, it turns into CCA evaporation of about $325 \mathrm{~s}$. Then it ends with mixed mode. However, for the case of $3.0 \mu \mathrm{l}$ water droplet evaporating on 20:1 PDMS surface, it exhibits a different behavior. The evaporation curves indicate that it has only two or three stages. In the first stage, the contact radius is no longer unaltered but decreases very slowly, at the same time the contact angle decreases. This stage can be called the weak CCR evaporation. The duration of this stage is about $625 \mathrm{~s}$. After a transition with shorter time as compared to evaporation of droplet on PDMS films with mass ratios of $5: 1$ and $10: 1$, it turns into CCA evaporation mode, however the droplet evaporates in the CCA mode for only about $100 \mathrm{~s}$. Then it switches into mixedmode evaporation. In other words, there is no evident CCA evaporation. The reductions in contact angle during CCR evaporation for evaporation of water droplet on PDMS films with mass ratios of $5: 1,10: 1$, and $20: 1$ are $5.16^{\circ}, 10.03^{\circ}$, and $27.51^{\circ}$, respectively. In a word, the higher the mass ratio of PDMS film, the longer the CCR evaporation and the shorter the CCA evaporation. 

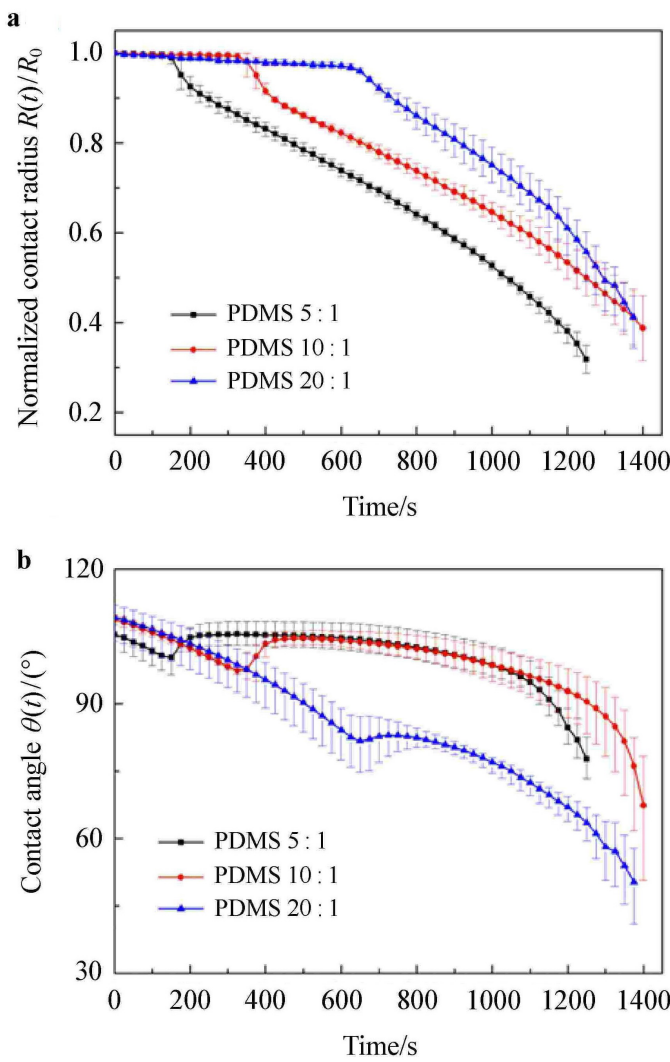

Fig. 5 Evaporation of water droplet on PDMS films. a Normalized contact radius; $\mathbf{b}$ Contact angle

From Fig. 5, we can easily find that PDMS films with different mass ratios have similar surface wettability, however, they have different evaporation characteristics, why? As we know, when there is a sessile water droplet on soft surface, there will be a ridge-like surface deformation under the resultant actions of vertical component of liquidvapor interfacial tension and Laplacian pressure. Such a deformation may be associated with molecular orientation of the solid molecules at the three phase contact line and thus might affect the evaporation characteristics, therefore, we simulate the deformation of PDMS films induced by sessile water droplet. The parameters are listed in Table 3, and the vertical displacement on the surface of PDMS films are shown in Fig. 6. The maximum heights for PDMS films with mass ratios of $5: 1,10: 1$, and $20: 1$ are respectively $74.7 \mathrm{~nm}, 80.9 \mathrm{~nm}$, and $152.4 \mathrm{~nm}$. Figure 6 indicates that the softer the film, the larger the deformation. As pointed out by Shanahan [36], the elastic stored energy per unit length of the three-phase contact line is proportional to $\gamma_{\mathrm{lv}}^{2}\left(1-v^{2}\right) / E$. Therefore, the higher the mass ratio of PDMS film, the more the elastic stored energy. As Lopes and Bonaccurso [31] said, such an energy acts as a barrier for the depinning of the three-phase contact line, hence the higher the mass ratio of PDMS film, the softer the film and the longer the CCR evaporation.
Table 3 Parameters for numerical simulation

\begin{tabular}{llll}
\hline Mass ratio & $5: 1$ & $10: 1$ & $20: 1$ \\
\hline$\theta_{0} /\left(^{\circ}\right)$ & 105.46 & 108.78 & 109.26 \\
$R_{0} / \mathrm{mm}$ & 0.97 & 0.94 & 0.93 \\
\hline
\end{tabular}

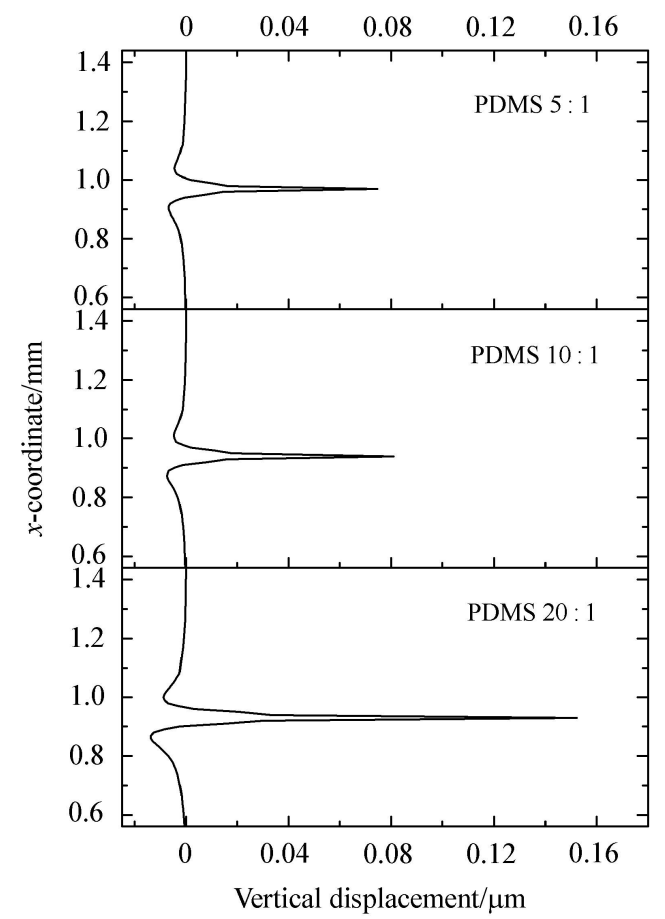

Fig. 6 Vertical displacement on the surface of PDMS films with different mass ratios

Finally, we make an order-of-magnitude estimation of the evaporation time for a water droplet, which is considered as a steady diffusion process. For simplicity and without loss of generality, we neglect the interaction between substrate and water droplet in the estimation of evaporation time. According to the Fick's first law of diffusion, the mass flux for an evaporating droplet is

$J=-D \nabla \rho$,

where $D$ is the diffusion coefficient of water and $\nabla \rho$ the density gradient. Thus, we can obtain an equation relating the mass changing rate of the water droplet to the density gradient as follows

$\frac{\mathrm{d} m}{\mathrm{~d} t}=\rho_{\mathrm{w}} \frac{\mathrm{d} V}{\mathrm{~d} t}=J S=-D S \nabla \rho$,

where $m$ is the mass of the droplet, $\rho_{\mathrm{w}}$ is the density, $V$ is the volume and $S$ is the surface area. With substitution of $V=4 \pi r^{3} / 3$ and $S=4 \pi r^{2}$ into Eq. (6), the following relation can be obtained to relate the radius changing rate to the density gradient 
$\frac{\mathrm{d} r}{\mathrm{~d} t}=-\frac{D \nabla \rho}{\rho_{\mathrm{w}}}$.

Integrating Eq. (7) from $t=0$ to $t=\tau$, we derived the evaporation time

$\tau=\frac{\rho_{\mathrm{w}} R}{D \nabla \rho}$,

where $R$ is the radius of the droplet at $t=0$. For a microliter droplet, the radius $R$ is of the order of $10^{-3} \mathrm{~m}$. By substituting $\rho_{\mathrm{w}} \sim 10^{3} \mathrm{~kg} / \mathrm{m}^{3}, D \sim 10^{-9} \mathrm{~m}^{2} / \mathrm{s}$, and $\nabla \rho \sim \rho_{\mathrm{w}} / R \sim$ $10^{6} \mathrm{~kg} / \mathrm{m}^{4}$ into Eq. (8), the characteristic time for water droplet evaporation should be: $\tau \sim 1000 \mathrm{~s} \sim 16 \mathrm{~min}$, which is of the same order as the measured evaporation time in the experiments. This order-of-magnitude estimation would assist our physical understanding on the evaporation of a water droplet.

\section{Conclusions}

We conducted the evaporation experiments of $3.0 \mu \mathrm{l}$ water droplet on PDMS films with different mass ratios, measured the Young's moduli of PDMS films using AFM and numerically calculated the surface deformation of PDMS films induced by sessile water droplet.

To conclude, with the increase of the mass ratio of PDMS film:

(1) Young's modulus of thin film is getting lower and the film becomes softer.

(2) Both surface deformation and the elastic stored energy are getting larger.

(3) The CCR evaporation mode of the droplet lasts longer in the entire evaporating period.

\section{References}

1 Feng, J.T., Zhao, Y.P.: Influence of different amount of Au on the wetting behavior of PDMS membrane. Biomed. Microdevices 10, 65-72 (2008)

2 Lim, K., Kim, S., Hahn, J.H.: Roller-type squeezing pump with picoliter handling capability. Sensor Actuat. B: Chem. 92, 208-214 (2003)

3 Takao, H., Miyamura, K., Ebi, H., et al.: A MEMS microvalve with PDMS diagram and two-chamber configuration of thermo-pneumatic actuator silicon. Sensor Actuat. A: Phys. 119, 468-475 (2005)

4 Byun, I., Yang, J., Park, S.: Fabrication of a new micro bio chip and flow cell cytometry system using Bio-MEMS technology. Microelectron. J. 39, 717-722 (2008)

5 Sodtke, C., Stephan, P.: Spray cooling on micro structured surfaces. Int. J. Heat Mass Tran. 50, 4089-4097 (2007)

6 Deegan, R.D., Bakajin, O., Dupont, T.F., et al.: Capillary flow as the cause of ring stains from dried liquid drops. Nature $\mathbf{3 8 9}$, 827-829 (1997)

7 Yunker, P.J., Still, T., Lohr, M.A., et al.: Suppression of the coffee-ring effect by shape-dependent capillary interactions. Nature 476, 308-311 (2011)
8 Dugas, V., Broutin, J., Souteyrand, E.: Droplet evaporation study applied to DNA chip manufacturing. Langmuir 21, 9130-9136 (2005)

9 Yuan, Q.Z., Zhao, Y.P.: Precursor film in dynamic wetting, electrowetting and electro-elasto-capillarity. Phys. Rev. Lett. 104, 246101 (2010)

10 Picknett, R.G., Bexon, R.: The evaporation of sessile or pendant drops in still air. J. Colloid Interf. Sci. 61, 336-350 (1977)

11 Wang, F.C., Zhao, Y.P.: Contact angle hysteresis at the nanoscale: A molecular dynamics simulation study. Colloid Polym. Sci. 291, 307-315 (2013)

12 Tadmor, R.: Approaches in wetting phenomena. Soft Matter 7, 1577-1580 (2011)

13 Chen, X.M., Ma, R.Y., Li, J.T., et al.: Evaporation of droplets on superhydrophobic surfaces: Surface roughness and small droplet size effects. Phys. Rev. Lett. 109, 116101 (2012)

14 McHale, G., Rowan, S.M., Newton, M.I., et al.: Evaporation and the wetting of a low-energy solid surface. J. Phys. Chem. B 102, 1964-1967 (1998)

15 Erbil, H.Y., McHale, G., Newton, M.I.: Drop evaporation on solid surfaces: Constant contact angle mode. Langmuir 18, 2636-2641 (2002)

16 Bourgès-Monnier, C., Shanahan, M.E.R.: Influence of evaporation on contact angle. Langmuir 11, 2820-2829 (1995)

17 Rowan, S.M., Newton, M.I., McHale, G.: Evaporation of microdroplets and the wetting of solid surfaces. J. Phys. Chem. 99, 13268-13271 (1995)

18 McHale, G., Aqil, S., Shirtcliffe, N.J., et al.: Analysis of droplet evaporation on a superhydrophobic surface. Langmuir 21, 11053-11060 (2005)

19 Yu, Y.S., Wang, Z.Q., Zhao, Y.P.: Experimental and theoretical investigations of evaporation of sessile water droplet on hydrophobic surfaces. J. Colloid Interf. Sci. 365, 254-259 (2012)

20 Hao, P.F., Lü, C.J., He, F.: Evaporation behaviors of water droplet on superhydrophobic surface. Sci. China: Phys., Mech. Astron. 55, 2463-2468 (2012)

21 Zhao, Y.P.: Physical Mechanics of Surfaces and Interfaces. Science Press, Beijing (2012) (in Chinese)

22 Shin, D.H., Lee, S.H., Choi, C.K., et al.: The evaporation and wetting dynamics of sessile water droplets on submicron-scale patterned silicon hydrophobic surfaces. J. Micromech. Microeng. 20, 055021 (2010)

23 Fang, X.H., Pimentel, M., Sokolov, J., et al.: Dewetting of the three-phase contact line on solids. Langmuir 26, 7682-7685 (2010)

24 Luo, C., Xiang, M.M., Liu, X.C., et al.: Transition from Cassie-Baxter to Wenzel States on microline-formed PDMS surfaces induced by evaporation or pressing of water droplets. Microfluid Nanofluid 10, 831-842 (2011)

25 Pericet-Cámara, R., Best, A., Butt, H.J., et al.: Effect of capillary pressure and surface tension on the deformation of elastic surfaces by sessile liquid microdrops: An experimental investigation. Langmuir 24, 10565-10568 (2008)

26 Pericet-Camara, R., Auernhammer, G.K., Koynov, K., et al.: Solid-supported thin elastomer films deformed by microdrops. Soft Matter 5, 3611-3617 (2009)

27 Yu, Y.S., Zhao, Y.P.: Elastic deformation of soft membrane with finite thickness induced by a sessile liquid droplet. J. Colloid Interf. Sci. 339, 489-494 (2009) 
28 Yu, Y.S.: Substrate elastic deformation due to vertical component of liquid-vapor interfacial tension. Appl. Math. Mech. 33, 1095-1114 (2012)

29 Liu, J.L., Feng, X.Q.: On elastocapillary: A review. Acta Mech. Sin. 28, 928-940 (2012)

30 Tadmor, R., Bahadur, P., Leh, A., et al.: Measurement of lateral adhesion forces at the interface between a liquid drop and a substrate. Phys. Rev. Lett. 103, 266101 (2009)

31 Lopes, M.C., Bonaccurso, E.: Evaporation control of sessile water drops by soft viscoelastic surfaces. Soft Matter 8, 38753881 (2012)

32 Dai, W., Zhao, Y.P.: The nonlinear phenomena of thin polydimethylsiloxane (PDMS) films in electrowetting. Int. J. Nonlinear Sci. Numer. Simul. 8, 519-526 (2007)
33 Chen, L., Auernhammer, G.K., Bonaccurso, E.: Short time wetting dynamics on soft surfaces. Soft Matter 7, 9084-9089 (2011)

34 Sun, Y.J., Akhremitchev, B., Walker, G.C.: Using the adhesive interaction between atomic force microscopy tips and polymer surfaces to measure the elastic modulus of compliant samples. Langmuir 20, 5837-5845 (2004)

35 Shanahan, M.E.R., Sefiane, K.: Kinetics of triple line motion during evaporation. Contact Angle, Wettability and Adhesion 6, 19-31 (2009)

36 Shanahan, M.E.R.: The influence of solid micro-deformation on contact angle equilibrium. J. Phys. D Appl. Phys. 20, 945950 (1987) 